\title{
Educadores militantes: Educar para la vida mediante el trabajo en empresas de inserción
}

\section{Educators and Activists: Educating for Life Through Work in Work Integration Enterprises}

\author{
Fernando Marhuenda Fluixá ${ }^{1 *}$ \\ Fernando.Marhuenda@uv.es \\ *Universitat de València, España
}

\section{Resumen:}

Este artículo se centra en el derecho a la educación para personas que viven en contextos de vulnerabilidad, en sintonía pero diferente a la aproximación de Escudero: en primer lugar, es una mirada sobre la educación no de jóvenes sino de personas adultas, muchas de ellas con trayectorias educativas truncadas, y todas ellas tras haber pasado por situaciones vitales que bien pueden calificarse de marginación y exclusión. En segundo lugar, ofrece una mirada a la práctica educativa en un contexto no escolar, sino productivo, ya que pone el foco de atención sobre el aprendizaje, tanto intencional como informal, que tiene lugar en empresas de inserción. En tercer lugar, nuestra perspectiva es la de los educadores, que en este caso no son docentes, sino personal de acompañamiento contratado en las empresas de inserción con la doble función de educar y promocionar al personal a su cargo a la vez que contribuir a la producción de la empresa.

A partir de tres casos cuya información se ha recogido y analizado cualitativamente, se exponen y discuten las funciones que realiza este personal, cómo planifican el itinerario y gestionan cada una de sus fa-

\section{Abstract:}

This paper explores the right to education for people living in contexts of vulnerability. The approach adopted is similar and yet different to that of Escudero's: first, we targeted adults instead of young people, many of them with a record of school failure and all of whom had gone through harsh life circumstances, close to marginalization or exclusion. Second, we approached educational practice out of school, within the context of production, focusing upon intentional and informal learning in work integration enterprises. Third, we took the view of educators, not teachers, life and job coaches working in work integration companies whose role was to educate and promote their staff as well as contributing to the company's production.

We drew upon three cases whose information was gathered and analyzed qualitatively. We described them and discussed their pedagogical role, how they planned the itineraries and managed the process, which competencies and knowledge they focused upon and, finally, and hence the title of the article, to what extent they understood their job as a life commitment which could be their educational

1 Dirección para correspondencia (correspondence address):

Fernando Marhuenda Fluixá. Dpto. de Didáctica y Organización Escolar. Universitat de València. Facultad de Filosofía y CC. De la Educación. Avda. Blasco Ibáñez, 30. 46010 Valencia (España). 
Educadores militantes: Educar para la vida mediante el trabajo en empresas de inserción

Fernando Marhuenda Fluixá

ses, en qué competencias y saberes ponen los énfasis y, por último, y de ahí también el título del artículo, en qué medida entienden su trabajo como un compromiso vital y cuál podría ser, por lo tanto, su enfoque de enseñanza, sin perder de vista las condiciones laborales ni a la precariedad en el mundo del trabajo.

\section{Palabras clave:}

Militancia; formación profesional; formación en el puesto de trabajo; competencias; aprendizaje informal; itinerarios de inserción. approach, without forgetting their working conditions and the precariousness of $\mathrm{cu}$ rrent labour markets.

\section{Key words:}

Activism; vocational education; on-thejob learning; competencies; informal learning; insertion itineraries.

\section{Résumé:}

Cet article se concentre sur le droit à l'éducation pour les personnes en situations de vulnérabilité, il s'agit d'une approche en harmonie mais différente de celle de Escudero: D'abord, c'est un regard sur l'éducation, non pas des jeunes mais des adultes, ayant des trajectoires éducatives tronquées et ayant vécu des situations vitales marginales et d'exclusion. Ensuite, un regard est lancé sur la pratique éducative dans un contexte qui $n^{\prime}$ est pas scolaire mais productif, vu que le centre d'attention est mis sur l'apprentissage aussi bien intentionnel qu'informel, qui a lieu dans les entreprises d'insertion. En troisième lieu, notre perspective est celle des éducateurs qui, en I'occurrence, ne sont pas des enseignantes, mais du personnel d'accompagnement employés dans les entreprises $d^{\prime}$ insertion avec le double rôle d'éduquer et de promouvoir le personnel sous sa responsabilité, ainsi que de contribuer à la production de l'entreprise.

A partir de trois cas, dont l'information a été recueillie et analysée de manière qualitative, les fonctions/rôles qu'exerce le personnel sont exposé(e)s et discuté(e)s ainsi que les compétences et les savoirs sur lesquels ils mettent l'accent et enfin, comme le titre I'indique, dans quelle mesure ils considèrent leur travail comme un engagement vital et, dès lors, quel pourrait être leur approche de l'enseignement sans perdre de vue les conditions de travail et la précarité dans le monde du travail.

\section{Mots clé:}

Militantisme; formation professionnelle; formation au travail; compétences; apprentissage informel; itinéraires $d^{\prime}$ insertion.

Fecha de recepción: 7-2-2018

Fecha de aceptación: 4-5-2018

\section{Introducción}

Pocas personas en la universidad española, en las ciencias de la educación, han escrito tanto como Juan Manuel Escudero sobre el derecho a la educación en contextos de vulnerabilidad y exclusión. Una problemática que ha abordado siempre en el contexto escolar, igual que otras a las que ha dedicado atención: innovación, cambio organizativo, desarrollo curricular o formación del profesorado. 
También este artículo se centra en el derecho a la educación para personas que viven en contextos de vulnerabilidad, pero con algunas diferencias respecto a la aproximación de Escudero: en primer lugar, es una mirada sobre la educación no de jóvenes sino de personas adultas, muchas de ellas con trayectorias educativas truncadas, y todas ellas tras haber pasado por situaciones vitales que bien pueden calificarse de marginación y exclusión. En segundo lugar, ofrece una mirada a la práctica educativa en un contexto no escolar, sino productivo, ya que pone el foco de atención sobre el aprendizaje, tanto intencional como informal, que tiene lugar en contextos empresariales y, en concreto, en empresas de inserción (Faedei y Aeress, 2014). En tercer lugar, la perspectiva del artículo es la de los educadores, que en este caso no son docentes, obviamente, sino personal de acompañamiento contratado en las empresas de inserción con la doble función de educar y promocionar a la vez que producir.

A partir de tres casos cuya información se ha recogido y analizado cualitativamente, se exponen y discuten las funciones que realiza este personal, cómo planifican el itinerario y gestionan cada una de sus fases, en qué competencias y saberes ponen los énfasis y, por último, y de ahí también el título del artículo, en qué medida entienden su trabajo como un compromiso vital (Casey, 1993), sin dejar de atender a sus condiciones laborales así como a la coyuntura de precariedad que caracteriza al mundo del trabajo hoy en día (Caballol, 2003).

\section{Marco teórico}

Esta contribución es parte de una investigación que analiza los procesos formativos y de acompañamiento que llevan a cabo las empresas de inserción, así como los contenidos formativos de esos procesos² ${ }^{2}$. En nuestro trabajo, tratamos de describir cuál es el funcionamiento de las empresas de inserción en tanto que organizaciones orientadas al aprendizaje para, de este modo, contribuir a identificar su valor social y educativo, más allá del estrictamente laboral.

En un contexto de creciente deterioro y precarización de las rela-

2 Proyecto de investigación financiado por el Gobierno de España: Procesos de formación, acompañamiento, cualificación y desarrollo personal en empresas de inclusión: Innovación en la inclusión social desde el empleo (EDU2013-45919-R). 
Educadores militantes: Educar para la vida mediante el trabajo en empresas de inserción

Fernando Marhuenda Fluixá

ciones laborales, nuestra investigación procura identificar relaciones entre cualificación, empleabilidad e inclusión social. El aprendizaje en el puesto de trabajo, con frecuencia informal y tácito, lo hemos empleado como marco de análisis para abordar estas relaciones (Hager \& Halliday, 2006; Stenström \& Tynjälä, 2009; Evans \& Waite 2010).

Las empresas de inserción son PYMEs que orientan su proceso productivo al tránsito al mercado de trabajo ordinario de sus trabajadores que se encuentran en proceso de inclusión social y cuyo contrato tiene por objeto el desarrollo de un itinerario personal de inserción, para el cual el empleo desempeña un papel central a la vez que instrumental. Se trata de empresas intencionalmente educativas.

Esto es posible gracias a la normativa que regula las empresas de inserción en España, que diferencia tres modalidades de contratación: personal técnico de producción (PTP), personal técnico de acompañamiento (PTA) y persona trabajadora en inserción (PTI). Son empresas de tránsito, orientadas a facilitar la salida hacia el mercado laboral ordinario: El contrato de inserción tiene una duración limitada de un año renovable a un máximo de tres años, y su objeto es el desarrollo de un plan individualizado orientado al desarrollo de competencias profesionales, sociales y personales, específicas para el puesto de trabajo objeto del contrato y transferibles a otros entornos laborales, recogidas en el anexo al contrato.

El trabajo educativo recibe en las empresas de inserción la denominación de acompañamiento, del que Quintao (2016) distingue tres tipos: acompañamiento sociolaboral, acompañamiento profesional e intermediación laboral. Como veremos a continuación, encontramos ejemplos de los tres en la práctica del personal de acompañamiento y producción.

A partir del estudio sobre los procesos de aprendizaje en el puesto de trabajo de Eraut y sus colaboradores (2007, 2009, 2012), que ha dado lugar al constructo de trayectorias de aprendizaje, se han identificado tipos de saberes a los que la experiencia en el puesto de trabajo puede dar lugar. Las trayectorias de aprendizaje indican qué se puede considerar progreso en el desempeño profesional de cada trabajador, en su sentido más amplio. Mediante una adaptación de la metodología de investigación de Eraut al contexto de las empresas de inserción, hemos utilizado este referente como marco de análisis empírico, lo que nos ha permitido aproximarnos a las personas en inserción como aprendices a la vez que al personal de acompañamiento y, en ocasiones, también al personal de 
producción, como facilitadores de sus procesos de aprendizaje y desarrollo personal, social y laboral.

\section{Marco empírico}

Nuestro trabajo es fruto de una trayectoria de estudio y colaboración con las empresas de inserción, iniciada en 2007 y consolidada a partir de 2010. A lo largo de este tiempo, en el marco de un convenio de colaboración y fruto de dos investigaciones con financiación pública, hemos tenido acceso a empresas en distintos lugares de España.

Nuestra investigación toma como referencia metodológica el trabajo de Eraut, que nos permite ofrecer descripciones de actuaciones bien valoradas por sus aspectos profesionales, técnicos u organizativos. En trabajos previos (Córdoba y Martínez, 2011; Marhuenda y Bonavía, 2011; Marhuenda, Navas y Bernad, 2010) hemos desvelado que hay algunos patrones comunes al desarrollo del aprendizaje y hemos apreciado indicios de procesos educativos en el puesto de trabajo de un segmento de la población que carece de cualificaciones formales (Navas y Marhuenda, 2013; Chisvert y Córdoba, 2017) y que encuentra en estas empresas la posibilidad de aprender en contextos laborales normalizados pese a su pasado de adicciones, medidas judiciales o dependencia de servicios sociales. Las trayectorias de aprendizaje son un heurístico útil para reconocer elementos del aprendizaje en el puesto de trabajo (Steadman et al., 2005). de producción y acompañamiento más vinculados a su itinerario.

Cada PTA y PTP ha participado en la investigación voluntariamente (al igual que las PTI de esas empresas), ha dado previamente su consentimiento para la obtención de sus datos, tanto en la observación como en la entrevista. En total, manejamos información de 24 profesionales de acompañamiento y producción que asumen tareas educativas en las empresas.

Hemos comunicado a cada empresa el protocolo que pensamos seguir a fin de asegurarnos que son conscientes de la demanda de información a lo largo del tiempo. Esto incluye toda la documentación de confidencialidad que sigue las directrices especificadas por el comité de ética en la investigación con humanos ${ }^{3}$ de la Universitat de València, y

3 http://www.uv.es/uvetica/index.html\# 
Educadores militantes: Educar para la vida mediante el trabajo en empresas de inserción

Fernando Marhuenda Fluixá

a las que los investigadores del resto de universidades se han adherido: Con cada empresa, firmamos un acuerdo así como un compromiso de cesión de datos para los propósitos de la investigación con cada uno de los trabajadores y trabajadoras observados y entrevistados, sea cual sea la naturaleza de su contrato. Además, facilitamos a la empresa información para conocimiento del resto del personal.

Acordamos con el personal de acompañamiento la fecha más adecuada para realizar el trabajo de campo, procurando estar presentes a lo largo de la jornada así como, en la medida de lo posible, en los protocolos de intercambio de información entre turnos, cuando corresponda. Las entrevistas tienen lugar tras la observación. La entrevista es semiestructurada y se ajusta según la fase del itinerario, pero hay preguntas que son fruto de la observación.

Este artículo muestra sólo algunos casos de personal de acompañamiento y producción que desempeña una función educativa que bien podríamos calificar como propia de educadores militantes, por utilizar la expresión de Escudero (2012).

Nuestra estrategia metodológica (Marhuenda, 2017) ha consistido en trabajo de observación de jornadas de trabajo completas del personal en inserción en distintas fases de su proceso, de las entrevistas con ese personal así como con personal de acompañamiento, de producción y de gerencia, hemos logrado obtener distintas descripciones empíricas, algunas de las cuales podríamos tipificar, como es el caso de los educadores militantes que aquí presentamos.

En el trabajo de campo, hemos puesto especial empeño en tratar de identificar los procesos formativos y de aprendizaje, el papel de los itinerarios de inserción en tanto que proyectos educativos individualizados, así como las relaciones educativas que se establecen en el seno de la empresa en un entorno que es productivo a la vez que tiene una pretensión inclusiva y educativa, en el que la evaluación de los aprendizajes está estrechamente relacionada con la corrección en el desempeño de las tareas del proceso productivo. Hemos prestado atención también a las estrategias organizativas y la cultura de la organización puesto que, a partir de trabajos previos (Molpeceres, 2004) somos conscientes de que organizaciones del Tercer Sector de Acción Social cuya misión es educativa procuran conformar equipos educativos que comparten los valores de las instituciones para las que trabajan.

Dado que nuestro estudio obedece a una estrategia longitudinal, si- 
guiendo los procesos de enseñanza y aprendizaje que experimentan personas con contratos de inserción a lo largo de su itinerario, los casos que ilustramos a continuación son de información recogida entre verano de 2015 y otoño de 2017. Según la disponibilidad de las empresas y nuestras posibilidades, el trabajo de campo ha tenido lugar en periodos no inferiores a seis meses y no superiores a un año, lo que nos ha posibilitado apreciar posibles cambios. En el trabajo de campo de los tres casos que se exponen en este artículo ha participado directamente el autor del mismo.

\section{Resultados}

Los resultados que presentamos a continuación son sobre las empresas en las que trabaja el personal de acompañamiento y producción cuyos casos exponemos a continuación, así como los principales rasgos del personal en proceso de inserción de cuya formación se encargan.

Tenemos también información sobre los tipos de actividades formativas que hemos identificado en cada uno de los casos, organizada según los criterios que nos proporciona el trabajo de Michael Eraut: procesos de trabajo con aprendizaje como efecto colateral, procesos de aprendizaje intencional y actividades de aprendizaje vinculadas a otros procesos. Así pues, de cada uno de los tres casos presentamos la información por empresas, modos de ejercer el acompañamiento, tipología de inserción y práctica de evaluación. Los casos han sido seleccionados para ilustrar aquí sólo aquellos que podríamos calificar de educadores militantes.

\section{Fundación Horizonte ${ }^{4}$}

Se trata de una empresa promovida por una Fundación con sede en distintas comunidades autónomas, de las más antiguas en el sector del reciclado, en cuya tradición se ha desarrollado.

Esta empresa tiene contratadas a personas adultas, casi todas con más de cuarenta y cinco años, por lo tanto ante una difícil inserción laboral en el mercado laboral ordinario. Tanto es así, que en el caso de una de las PTI, la expectativa es la de llegar a la edad de jubilación dentro de

4 Este nombre, como el resto de nombres propios que aparecen en el artículo, es ficticio. 
Educadores militantes: Educar para la vida mediante el trabajo en empresas de inserción

Fernando Marhuenda Fluixá

esta empresa. Se trata de personas con problemas personales, afectadas por sucesos de distinto tipo (laboral, familiar, relaciones con la justicia, adicciones) que han alterado su curso vital ya en la edad madura, y que sin embargo tienen experiencia laboral previa en distintos sectores profesionales, en todos los casos con más responsabilidad de la que pueden desempeñar ahora, así como también con un mayor nivel de cualificación que el del puesto de trabajo que actualmente ocupan. Una de ellas se ve como simple trabajadora y no tiene mayor expectativa de acompañamiento ni conciencia de encontrarse en un proceso de inserción, las otras tres PTI de la empresa valoran el trabajo de apoyo personal que están recibiendo en la empresa.

La empresa tiene sede en dos poblaciones cercanas, ambas de tradición industrial, pero no es la única empresa en este sector en el territorio, por lo que percibe a otras como posible amenaza.

La gerencia de la empresa toma las decisiones sobre las PTI aunque en algún caso ni PTA ni PTP las compartan.

La PTA tiene responsabilidades sobre la gestión de la formación y la intermediación que realiza la entidad, y tiene alguna dificultad para encontrar el momento (así como la escucha) por parte de alguna de las PTI, que no reconoce la contribución que puede suponer en su proceso. Pero es la PTP la persona que hemos escogido para presentar en este artículo. Le Ilamaremos Santi. Con estudios obligatorios completos, Santi vive una vida convencional, casado, con hijos. Antes de trabajar en esta empresa, en la que lleva aproximadamente una década, tuvo otros trabajos, el inmediatamente anterior fue de encargado de reparto de alimentos. Además, se ha dedicado profesionalmente a la música, aunque nunca en exclusividad, porque los ingresos no lo permitían. En su tiempo libre, es también monitor de cursos de formación online. Su paternidad, igual que su dedicación como monitor, así como, desde luego, su dedicación profesional actual, los vive como una vocación educativa.

Santi es una persona paciente, observadora, atenta, que gusta de escuchar a la gente. Tiene sus propias ideas, unos principios de actuación claros, que va desgranando en las entrevistas no sólo mediante ejemplos de cuáles son sus prácticas, sino argumentando y dando razón de porqué toma las decisiones que toma y cuáles son las justificaciones que, a partir de la experiencia, la propia y la vicaria, le han llevado a ir escogiendo modos de hacer más efectivos.

Entre sus principios destaca conocer bien a la persona a la que ha de 
acompañar, no prejuzgarla, entenderla, puesto que confía en la capacidad de todas sus PTI para desarrollarse como personas y como profesionales. Esta misma convicción es la que le lleva a corregirles cuando lo estima oportuno, algo que acostumbra a hacer no de inmediato sino dejando que transcurra un tiempo para conseguir que la persona esté en mejor disposición de escuchar y aprender de su evaluación.

Podemos destacar varios elementos de su forma de educar: Por una parte, reconocer cuando la PTI está en disposición o no de escuchar y con voluntad de aprender, para escoger el momento propicio. Ese momento pasa por la oportunidad de trabajar conjuntamente, de dedicar varios días seguidos a compartir el trabajo, ya sea en el almacén, en la tienda o en el camión de recogida. Y al compartir ese trabajo, lo primero que hace Santi es dar ejemplo con su propia práctica, poniéndose manos a la obra, sin explicar, dando apenas alguna indicación sobre la forma más operativa de llevar a cabo la tarea. Santi tiene claros los objetivos de producción, también los de acompañamiento, sabe que los primeros tienen plazos más cortos que los segundos, y sabe que el trabajo bien hecho da resultados positivos aunque haya que esperar a verlos. A instancias de las PTI, sí que ofrece sus recomendaciones de trabajo, si bien prefiere invitar a cada PTI a reflexionar sobre el desarrollo de la tarea, más que sobre el propio desempeño, teniendo en cuenta que el fruto del trabajo en esta empresa es siempre fruto del esfuerzo colectivo. De este modo, traslada la responsabilidad a cada persona en lugar de asumirla él por el resto, en una invitación al trabajo en equipo.

Cuando valora que es buen momento, le cede la iniciativa en la realización del trabajo a la PTI, para forzarla a tomar decisiones, a explicarlas, para permitirle también equivocarse cuando prevé que esa situación va a poder dar lugar a algún aprendizaje significativo. Aprender de la experiencia es el pilar fundamental de la práctica educativa de Santi, una experiencia en la que el error y la equivocación tienen cabida, y en la que se han de justificar las decisiones que se toman. En ocasiones, delega en alguna PTI la tarea de instruir a algún miembro nuevo del equipo.

Respecto a la organización del trabajo, Santi procura mantener a cada pesona en un mismo puesto, aquél en que la persona se encuentra más capacitada y a gusto, pero intenta también que haya una mínima rotación, más que para aprender del desarrollo del puesto para que cada cual tenga conocimiento de la carga de trabajo que tiene cada miembro de la plantilla, con sus exigencias y a veces con sus propias penalidades. 
Educadores militantes: Educar para la vida mediante el trabajo en empresas de inserción

Fernando Marhuenda Fluixá

Estas rotaciones son siempre aprovechando las necesidades de producción que tiene la empresa, por lo que su función educativa queda siempre en segundo plano.

En el trabajo diario y el contacto con el día a día, en la disponibilidad de Santi para hacer más rotaciones por puestos que el resto de la plantilla, aprovechando así la oportunidad de trabajar con distintas PTI, se encuentra la posibilidad de realizar el acompañamiento de forma cotidiana y sostenida en el tiempo, así como de ir planificando tareas apropiadas para el progreso y satisfacción de las PTI así como la capacidad de espera.

Esa capacidad de espera, propia del trabajo educativo, Santi la manifiesta dejando a cada PTI ser ella misma, no pretendiendo imponer una forma de ser ni una forma de hacer, pero sí dentro de una norma: la de trabajar diligentemente, eficientemente, amablemente, pensando en los destinatarios del trabajo, ya sea una familia que desea vaciar un piso, un almacén organizado y limpio, la limpieza de los productos que se ponen a la venta o el aseo del personal en el desempeño de su puesto de trabajo. Para Santi, el trabajo es honesto y dignifica, y la actitud y la disposición con la que se afrontan son importantes. Es consciente, y trata de transmitirlo, que se trabaja con objetos que son de personas y para personas, y que el respeto a unas y otras es importante, como también lo es el respeto entre los miembros del equipo de trabajo.

Para finalizar, cabe señalar que a Santi le gustaría tener más comunicación con la PTA y que sus valoraciones fueran tenidas algo más en cuenta, sin que caiga en cuestionar el trabajo que realizan ni en minusvalorar su ocupación ni capacitación. Es consciente de su lugar en la empresa, de las responsabilidades que le corresponden y de las obligaciones a las que debe atender. Podríamos resumir diciendo que su militancia es educativa, y que su trabajo educativo forma parte de su actitud ante la vida, de ayuda, apoyo y facilitación, de amabilidad, de confianza, de esperanza. En este sentido, realiza su trabajo como tantas educadoras infantiles, poniendo especial empeño en disponer el espacio y aprovechar las oportunidades, relegando el curriculum a un segundo plano para anteponer las necesidades e intereses de las personas cuya formación tiene a cargo, se interesa por ellas sin entrometerse en su vida, las valora como personas y no sólo como compañeras de trabajo. Es un mentor, nada invasivo en su tarea educativa, pero siempre oportuno a la hora de responder a las demandas que se le plantean. 


\section{Asociación La Parada}

Esta empresa acaba de registrarse como empresa de inserción, después de una trayectoria de casi tres décadas como empresa autogestionada por una asociación de personas paradas que se movilizaron para buscarse empleo a mediados de los años ochenta. Trabaja en el sector de reciclado y venta de segunda mano.

Las tres PTI son mujeres, una de ellas ya ha trabajado en otra empresa de inserción de la población en el mismo sector, es una persona joven sin mayores expectativas respecto a formación ni carrera, ni mayores problemáticas vitales. Las otras dos son mujeres de más de 40 años que han sido víctimas de violencia de género, ambas con hijos a su cargo.

La PTA es también una mujer joven que ejerce también como PTP y se ha incorporado recientemente a la empresa. Hay otras dos PTP, fundadoras de la asociación, que trabaja cada una en la tienda de la que es responsable. Tanto PTA como PTP tienen recursos formativos y personales, vinculados tanto a su trayectoria profesional como a su propio interés en el crecimiento y desarrollo personal, algo que es muy bien valorado por las PTI.

Mariola es la persona en la que nos vamos a centrar. Desempeña el roles de PTA pero también ejerce de PTP todos y cada uno de los días, un trabajo que realiza con entusiasmo, soltura y rigor. Con formación universitaria específica, a diferencia del resto de la plantilla, Mariola tiene un conocimiento técnico profundo del conjunto de procesos de trabajo de la empresa. Mariola, que vive con su pareja y disfruta de la Naturaleza, es una persona muy ocupada. Además de su trabajo en la empresa, da clases particulares, realiza ejercicio varios días a la semana, y ha realizado estudios de máster y tiene intención de cursar un doctorado vinculado a su experiencia profesional pero sobre todo a su activismo político, que se sitúa en el ámbito de la economía alternativa y solidaria: participa en varias redes en alguna de ellas representando a la empresa pero principalmente por su propio interés.

Desde el principio de nuestro trabajo de campo en la empresa hemos apreciado un énfasis muy nítido en los procesos educativos, que van desde la mejora del desempeño en el puesto de trabajo a la promoción de la alfabetización básica, así como también el acompañamiento social, tanto en la gestión de problemas de la vida cotidiana como en el trabajo sobre circunstancias personales que han afectado seriamente a la vida 
Educadores militantes: Educar para la vida mediante el trabajo en empresas de inserción

Fernando Marhuenda Fluixá

de estas mujeres. Hay, además, un empeño por proporcionar una lectura crítica del mundo y del entorno más cercano, próximo al compromiso activo en movilizaciones sociales: Mariola está convencida de que estas mujeres no sólo deben hacer frente a sus circunstancias personales sino que lo que les sucede es fruto de la organización social, y por eso desea que luchen por sí mismas pero que compartan criterios. Su trabajo es de emancipación personal y de toma de conciencia colectiva. Todo ello, sin menoscabo de procurar momentos de esparcimiento y celebración, tanto cotidianamente como en momentos señalados.

Mariola realiza su trabajo de acompañamiento tratando de seguir las orientaciones que ofrece el manual publicado por Faedei y Aeress (2014). Esto supone que, en primer lugar, se traza una planificación del acompañamiento, con objetivos a alcanzar por cada una de las PTI, a corto, medio y largo plazo. Son objetivos que ella tiene claro, aunque prefiere no trasladarlos a las PTI, para no presionarlas. Pero son objetivos que guían su acción educativa y su modo de proceder, y que tienen mucho que ver con reforzar la autoestima y promover el desarrollo personal y la autonomía de esas mujeres.

Está muy atenta a las circunstancias personales y familiares que afectan a las PTI, podría parecer que incluso excesivamente, puesto que es conocedora de sus situaciones y problemas y les ofrece su apoyo, que no siempre limita al espacio y tiempo de la jornada laboral. Es conocedora y en ocasiones incluso participa de visitas a Servicios Sociales, a la Unidad de Conductas Adictivas, de las gestiones para el alquiler del piso o de las reuniones escolares. Es significativo, también, el apoyo que ofreció para que una de estas mujeres pudiera disfrutar de las primeras vacaciones en su vida.

Muestra un empeño mayor que el que hemos podido observar en otras empresas de inserción en conseguir que las PTI se matriculen en la escuela de personas adultas y traten de obtener el Graduado en Educación Secundaria Obligatoria. Tan relevante lo considera que dedica un tiempo cada día, dentro de la jornada laboral, a trabajar con ellas sobre lo que van haciendo en la escuela, estando al día de sus tareas y de sus progresos, facilitándoles también recursos para el aprendizaje.

Aprovecha los recursos a su alcance, por ejemplo los tecnológicos, como un grupo de whatsapp para mantener contacto diario con todas las personas a su cargo, incluso cuando están en otros centros de trabajo de la empresa, así como para animar y estimular y generar clima positivo 
de grupo; también en alguna ocasión ha utilizado vídeos para exponer en youtube donde ha conseguido implicar al personal en un trabajo de expresión en equipo. Además, hace uso de la organización del espacio, en la nave industrial, de modo que desempeña su labor al tiempo que puede observar cómo realizan su trabajo las mujeres que acompaña, lo que le proporciona claves para el trabajo individual de acompañamiento. Además, introduce y estimula momentos de descanso que, si bien son breves, procura hacer en grupo en torno a alguna chocolatina, dulce o cualquier otra excusa que sirve para tener momentos de satisfacción y relajación, de distensión, sin que por ello deje de lado el objetivo de producción que la empresa tiene marcado y que alcanza con esfuerzo pero sin problemas. Se preocupa por generar un buen ambiente aunque también ha tenido que lidiar con alguna situación complicada de relaciones entre trabajadoras.

Estimula a las mujeres a asumir los errores y aprender de ellos, aunque es consciente de que en situaciones de baja autoestima esto resulta especialmente complicado, así como que algunas circunstancias vitales de estas personas invitan a esconder información, lo que da lugar a alguna situación de desconfianza que, con el tiempo, se ha visto fundada.

La comunicación es un elemento clave en el trabajo de Mariola, que la utiliza tanto como mecanismo de relación personal como instrumento para mejorar la capacidad de comprensión del mundo y de expresión de las emociones, frustraciones, ilusiones, así como para hacer frente a los conflictos de cara. Esto conlleva que la relación que establece Mariola con las PTI desdibuje su rol jerárquico, algo que sin duda ha contribuido a ganarse la confianza de las mujeres desde los inicios de su trabajo, al igual que el hecho de que el acompañamiento lo realice principalmente desde el desempeño de su puesto de trabajo de producción, como una trabajadora más que no tiene despacho sino que está al servicio de las necesidades diarias de la empresa. En contrapartida, quizá estas mujeres no tienen una conciencia clara de estar participando en un proceso de inserción y, sin embargo, conforme pasa el tiempo parece que Mariola lo va consiguiendo.

\section{Empresa En marcha}

La empresa está promovida por una orden religiosa que a su vez tiene una buena red de contactos entre sus clientes. Es la única empresa pro- 
Educadores militantes: Educar para la vida mediante el trabajo en empresas de inserción

Fernando Marhuenda Fluixá

movida por la entidad, que tiene muy claro que está en este negocio por la acción social que supone, no por el negocio en sí ni por generar beneficio económico. Está ubicada en un polígono industrial, y la empresa está valorando en ampliar su línea de negocio industrial para incorporar nuevos servicios, de modo que pueda disponer de más puestos de trabajo. La competencia es dura, especialmente por parte de las empresas que trabajan para otra de las grandes organizaciones no gubernamentales.

La empresa contrata a personas de ambos sexos, en su mayor parte de origen inmigrante, y tiene una PTA que realiza también diariamente funciones de PTP. La PTP principal es una de las primeras PTI que hubo en la empresa, y que culminó con éxito su itinerario. La gerente también realiza tareas tanto de producción como de acompañamiento.

El caso que hemos escogido es el de Juana, con formación universitaria en el ámbito de las ciencias sociales, una buena relación con miembros de la orden religiosa con quienes comparte valores. Por su formación, tiene muy claros los principios de actuación y también el tipo de personas con las que trabaja, algunas de las cuales han atravesado procesos vitales de gran violencia, y con las que es exquisita en el trato. Pero también es consciente de que está tratando con personas que ahora son trabajadoras, y que pese a que deben seguir sanando heridas del pasado, han dejado ya de ser víctimas y se encuentran en un proceso de reconstrucción personal.

Juana se muestra siempre sonriente y afable, con una sonrisa franca, está pendiente de la gente pero tiene el horizonte de su trabajo muy claro: capacitar a sus compañeras, profesional, social y emocionalmente, ayudarles a ser independientes, facilitar las condiciones para que puedan elegir el tipo de vida que quieren llevar, conseguir que sus circunstancias se normalicen para que ellas puedan así tomar el control de su vida. Esto ha dado lugar en alguna ocasión a que Juana proponga, de acuerdo con la gerencia, acortar los plazos del proceso de inserción en beneficio de la propia PTI, algo que otras empresas no se plantean, agotando los plazos previstos por la legislación.

El trabajo es el eje del trabajo de Juana, que disfruta de la doble condición de acompañante y peón de producción para conocer bien qué le puede pedir a cada PTI en cada momento y cómo plantearles retos que puedan ir superando con el paso del tiempo. La organización del trabajo es propicia para este propósito, ya que hay distintos puestos en la empresa por los que hacen pasar a todo el personal, con la ventaja añadida 
de que todos ellos están en el mismo espacio físico. La productividad, la eficacia en el desempeño de la tarea, el trabajo bien hecho y acabado, la conciencia del trabajo realizado, son todos ellos elementos cruciales en los procesos formativos de la empresa. La plantilla sabe que no basta con hacer el trabajo, que hacerlo a tiempo y hacerlo bien es un requisito indispensable, sea quien sea el cliente para el que estén trabajando, y estas condiciones son de cumplimiento obligado incluso aunque se trabaja bajo la presión del tiempo la mayoría de jornadas. A diferencia del caso anterior, la concentración es necesaria para realizar este trabajo, lo que lleva a realizarlo muchas veces en silencio, con la comunicación precisa para el desempeño de las tareas, si bien habitualmente se trabaja con música de fondo (que también sirve para amortiguar el ruido de la maquinaria industrial).

Pese a compartir con el resto del personal el trabajo de producción y tener los descansos en el mismo momento, Juana sabe que el trabajo de acompañamiento lo hace en su despacho, en entrevistas individuales que invitan a la persona a abrirse. En este sentido, hace uso de un diario de acompañamiento que es una herramienta que no hemos visto utilizar en otras empresas y que, sin embargo, le permite llevar un registro de progresión a lo largo del tiempo y planficar sus intervenciones adecuadamente. Para ello, además, mantiene una relación fluida con otras entidades y servicios sociales, pero plenamente consciente de que su trabajo es en torno a los procesos productivos y las relaciones laborales que tienen lugar en la empresa. Sin embargo, tiene conciencia de no atender los procesos de acompañamiento con la intensidad y frecuencia que desearía, porque la producción es la que marca el ritmo de todos los procesos.

El trabajo de acompañamiento lo lidera Juana, pero se apoya en el trabajo de otras dos compañeras, la PTP, que fue una de las primeras trabajadoras de inserción en la empresa y que desempeña el papel de referente y responsable de producción, con exigencia y rigor pero con la autoridad que le reconoce el conjunto de la plantilla; así como con la gerente, quien también cuenta con esa autoridad por su compromiso, dedicación, su humanidad y su honestidad. También Juana reúne estos rasgos y cuenta con el reconocimiento de toda la plantilla, mujeres y hombres, muchos de ellos de más edad que ella. Todo esto le permite aplicar una rendición de cuentas que es mucho más visible que en otras empresas y que es bien aceptada por la plantilla que, en su mayor parte, se encuentra toda ella en proceso de inserción. 
Educadores militantes: Educar para la vida mediante el trabajo en empresas de inserción

Fernando Marhuenda Fluixá

\section{Discusión}

Iniciamos esta discusión de los resultados expuestos, algunos de los cuales ya hemos ido comentando en la sección anterior, en torno a las funciones del personal de acompañamiento y producción y los procesos de formación que llevan a cabo, siguiendo el esquema planteado por Eraut y descrito más arriba.

Respecto a los procesos de trabajo con aprendizaje como efecto colateral, podemos afirmar que los tres educadores que acabamos de retratar realizan un trabajo individualizado en su acompañamiento pero procuran favorecer el trabajo en equipo para el desarrollo de los procesos productivos, que son los que los tres toman como eje. Entienden el trabajo como una actividad que no se realiza en solitario, enseñan que el producto de su trabajo es fruto de todas las personas que han participado en el proceso productivo, y procuran poner atención en el respeto a los colegas de trabajo y a su contribución a cada una de las tareas.

La organización de grupos parece responder más a necesidades de la producción que a un propósito formativo, y en los tres casos se combina la especialización en un puesto con el conocimiento de los otros puestos, poniendo también el énfasis en la capacidad de colaborar por encima de las diferencias personales: el trabajo tiene una dimensión colectiva.

En los tres casos la persona que realiza el acompañamiento participa también del proceso productivo. Es una persona que conoce los pormenores del trabajo diario, que suple con su productividad la que pueda faltar en las personas de inserción a las que acompañan, que les enseña con su ejemplo y que disfruta de su trabajo, que en todos los casos entienden como servicio, valorando la utilidad que tiene para la clientela a la que atienden.

El gusto por el hecho de trabajar se une también a la sintonía con los valores de la empresa, a compartir la filosofía que la mueve y que se traduce en la creación de un entorno de trabajo acogedor y amable. Además, en los tres casos se muestran disponibles para atender a las necesidades y demandas de las personas que acompañan, y en ninguno de los tres tratan de imponer ni forzar procesos, mostrando una paciencia destacable. Muchas de las demandas, incluso las que tienen que ver con el desarrollo personal y social, son satisfechas en el mismo proceso de desempeño laboral. El trabajo se convierte así en un elemento humani- 
zador y, por lo tanto, en el principal factor normalizador de la vida de estas personas.

Los tres educadores buscan asignar tareas que puedan suponer un desafío a las personas a las que acompañan cuando valoran que se encuentran en condiciones de poder responder al mismo y no bloquearse, aunque el bajo nivel de cualificación de alguna de las actividades que desempeñan no siempre permite esto. La baja cualificación, el carácter rutinario de muchos de los puestos y la dinámica habitual de trabajo tienen como contrapartida que evitan la aparición de problemas. No hay indicios de participación en innovaciones ni de oportunidades de consolidar y mejorar destrezas que ya se tienen, y hay conciencia del trabajo con clientes, aunque no se potencie especialmente.

Si prestamos atención a los procesos de aprendizaje intencional, en los tres casos podemos afirmar que hay una supervisión efectiva, no intrusiva, no directiva, facilitadora de procesos de aprendizaje entre personas adultas. Se trata de una supervisión, además, que está centrada en procesos productivos pero con una orientación hacia el desarrollo personal y social de las personas en inserción, que se articula así en torno al proceso productivo, lo que resulta viable gracias a la asunción de funciones de acompañamiento y producción, siendo esta última fuente de reconocimiento y autoridad.

No apreciamos indicios de promover observación de puestos de trabajo ajenos, más allá de las rotaciones organizadas con esta finalidad y siempre cuando el ritmo de la producción lo aconseja. Sí que hemos apreciado en la segunda de las empresas que se promueve la participación en actividades formativas para las que la educadora se apoya en la EPA, pero ninguno orientado a la obtención de una cualificación profesional, si bien alguna de estas tres empresas dispone de certificación de referencia en el Catálogo Nacional. En los tres casos, además, se atiende a los intereses formativos que manifiestan los trabajadores en inserción.

Tanto Juana como Mariola y Santi son ejemplos de la gestión pausada y planificada de los procesos de inserción en las empresas, y así lo reconocen las PTI a las que acompañan. La gestión del itinerario es planificada, personalizada y ajustada al ritmo de cada una de ellas, sabiendo cuándo proponerles retos y exigirles. Sin embargo, las tres, especialmente Juana, que es quien tiene la formación más indicada para llevar itinerarios de inserción, desearían poder atender con más intensidad a los itinerarios, que quedan a expensas de los ritmos de producción de 
Educadores militantes: Educar para la vida mediante el trabajo en empresas de inserción

Fernando Marhuenda Fluixá

las empresas. Esto, sin embargo, lo tornan en un elemento educativo, pues le conceden al trabajo el papel central que todavía tiene en la vida adulta.

Nos centramos finalmente en las actividades de aprendizaje incorporadas a otros procesos. Santi, Mariola y Juana tienen una gran capacidad de escuha y observación, cualidades que procuran que desarrollen las PTI, y hacen un uso inteligente de las preguntas en sus procesos de acompañamiento personal. Cuentan para ello con el respaldo de la gerencia de sus empresas. Observar y preguntar son actividades que forman parte del día a día y que dependen de lo que la persona que observa y escucha es capaz de asimilar y comprender.

Por la forma de realizar el trabajo, los tres se han convertido en referentes y también en las personas a las que buscan las PTI cuando precisan de información o de recursos. En todos los casos, las PTI tienen claro a quiénes acudir, a quiénes preguntar, quiénes son las personas que ocupan puestos de responsabilidad que en estos tres casos coinciden con las personas dispuestas a ayudar; se les reconoce la capacidad y la voluntad de apoyo. Además, en tanto que participan de los procesos productivos, dan feedback de forma inmediata en todo lo que se refiere al desempeño del trabajo, salvo Santi, que prefiere realizar las correcciones sin estar en presencia de clientes y a la espera de un momento propicio para que la PTI encaje bien la corrección. Esta evaluación informal encaja bien dentro de los procesos de aprendizaje informal y el propio itinerario de inserción avanza mediante la adquisición de conocimiento tácito. La evaluación sigue la misma tónica de informalidad que el resto de procesos formativos y de acompañamiento, es más incidental que planificada, y apenas deja evidencias registradas.

Si bien no hemos encontrado herramientas específicas para la supervisión de los procesos, más allá de los diarios de seguimiento, los tres casos proporcionan evidencias abundantes de criterios profesionales propios, razonados, basados en una combinación de experiencia y formación, y referidos en todos los casos, de una u otra forma, a las reflexiones y pautas que figuran en el manual de acompañamiento de Faedei y Aeress (2014). 


\section{Conclusión}

Juan Manuel Escudero (2012) define al profesor militante como aquel que reúne tres tipos de fortaleza: intelectual (por su talante, sin buscar certezas donde no las haya pero apasionado por el conocimiento), emocional (una persona afectiva, capaz de valorar la vivencia de las tareas y no sólo su quehacer, de darle significación personal a la relación y humanidad a la profesión), así como moral (con sentido de pertenencia a alguna ideología y primando por encima de todo el reconocimiento de los derechos de las personas y, en concreto, del derecho a la educación). Bien podemos decir que los tres casos que hemos documentado reúnen estas tres cualidades en la fortaleza con la que Ilevan adelante su trabajo.

Somos conscientes, además, de que detrás de su profesionalidad y su humanidad hay un trabajo colectivo, una vocación de servicio compartida, que ha sido puesta de manifiesto, entre otras, en el manifiesto ${ }^{5}$ suscrito en El Escorial en noviembre de 2013, que da muestra de lo que podríamos calificar como inteligencia colectiva y pública (Escudero, 2012). Nos atrevemos a afirmar que nos encontramos ante tres casos de educadores que son formadores, acompañantes y mentores. Educadores que tienen conciencia de serlo y vocación, que disfrutan de su trabajo.

Saben el valor de su trabajo, saben el valor del trabajo, saben que están ante procesos de reconstrucción personal, de enriquecimiento cultural, de promoción de autonomía personal y toma de conciencia. Su trabajo tiene un componente pedagógico visible, hacen uso de elementos propios del trabajo tales como la postura física de las PTI, la gestión de los espacios, la organización del trabajo en equipo, la búsqueda de recompensas y momentos de desenfado, el establecimiento de metas concretas y, como elemento común a los tres casos, una ética del trabajo que incluye tanto el cumplimiento de las obligaciones como la aspiración a realizar un trabajo bien hecho. En este sentido, estos militantes educativos podrían también calificarse de militantes obreros, al estilo de lo señalado por Gil (2017) o Teitelbaum (2017).

Generan posibilidades de aprendizaje en las PTI planificando su trabajo, delegando, explicitando los objetivos en materia de producción. También se muestran abiertas y confiadas en las capacidades de las PTI con las que trabajan, y dan muestras de cercanía personal, siempre desde el respeto a la otra persona y garantizando la confidencialidad, pero

5 http://www.faedei.org/images/docs/documento41.pdf 
Educadores militantes: Educar para la vida mediante el trabajo en empresas de inserción

Fernando Marhuenda Fluixá

sin comprometer el desempeño profesional. No tienen reparo en buscar momentos para la formación y la atención personal, ya sea previéndolos en la carga de trabajo diaria o bien habilitándolos en el quehacer habitual. Son conscientes de que trabajan re-educando a personas adultas, y es el ritmo de la persona, más que la fase en la que se encuentra, lo que marca la pauta de su trabajo educativo, de nuevo un ejemplo de lo que Escudero (2013) ha denominado comprensión ecológica del riesgo, y que les lleva a trabajar sobre las distintas dimensiones de exclusión que padecen.

\section{Referencias bibliográficas}

AERESS y FAEDEI. (2014). El acompañamiento en las Empresas de Inserción. Madrid: Faedei/Aeress.

Caballol, J.M. (coord.)(2003). De la exclusión al empleo. Nuevos espacios de participación ciudadana. Madrid: RAIS.

Casey, K. (1993). I answer with my life: Histories of women teachers working for social change. New York: Routledge.

Chisvert, M.J. \& Córdoba, A. (2017). Validation and accreditation of qualifications and citizenship: a method to guarantee social equity in Spain. En F. Marhuenda. Vocational education beyond skill formation: VET between civic, industrial and market tensions. Bern: Peter Lang, 319-342.

Córdoba, A.I. y Martínez, I. (coords.) (2011). Trabajo, empleabilidad y vulnerabilidad social. Valencia: PUV.

Eraut, M. (2009). Improving the quality of placements. AERA annual conference, San Diego.

Mckee, A. \& Eraut, M. (eds.)(2012). Learning trajectories, innovation and identity for professional development. Dordrecht: Springer.

Eraut, M. \& Hirsh, W. (2007). The significance of workplace learning for individuals, groups and organisations. Oxford: SKOPE Monograph 9.

Escudero, J.M. (2012). Militantes. En J. SÁEZY J. GARCÍA (cords.) Metáforas del educador. Valencia, Naul Llibres, 113-120.

Escudero, J.M. (coord.)(2013). Estudiantes en riesgo, centros escolares de riesgo. Respuestas educativas al alumnado en situaciones de vulnerabilidad. Murcia, Diego Marín.

Evans, K. \& Waite, E. (2010). Stimulating the innovation potential of routine workers through workplace learning. European Review of Labour and Research, 16(2), 243258.

Gil, G. (2017). Education and work in the libertarian thinking of $19^{\text {th }}$ and early $20^{\text {th }}$ century Spain. En F. Marhuenda. Vocational education beyond skill formation: VET between civic, industrial and market tensions. Bern: Peter Lang, 139-162. 
Educadores militantes: Educar para la vida mediante el trabajo en empresas de

inserción

Fernando Marhuenda Fluixá

Hager, P. \& Halliday, J. (2006). Recovering informal learning. Wisdom, judgement, community. Dordrecht: Springer.

Marhuenda, F. (2017). Learning at work: Researching personal development and competence building in work integration companies. Educar, 1-19.

Marhuenda, F. y Bonavía, T. (2011). Estrategias de formación en las Els: Aplicación del modelo de trayectorias de aprendizaje de Eraut. En A. Córdoba \& I. Martínez (coords.). Trabajo, empleabilidad y vulnerabilidad social. Valencia: PUV, 183-227.

Marhuenda, F., Bernad, J.C, \& Navas, A. (2010). In-company work experience as a strategy for educating and inserting people in the labour market: work integration social enterprises. Revista de Educación, 351, 139-161.

Navas, A. Y Marhuenda, F. (2013). Transitions from exclusion to Work Integration Social Enterprises and into the ordinary labour market. En J. Seifried \& E. Wuttke (eds.). Transitions in Vocational Education, Research in Vocational Education, 163-184. Opladen: Barbara Budrich.

Molpeceres, M.A. (2004). Identidades y formación para el trabajo. Montevideo: CINTERFOR/OIT.

Quintao, C. (2016). The marketing and coaching functions in WISE. Exploratory study in 5 European countries. EPP project. Unpublished document.

Steadman, S. Et Al. (2005). Methodological challenges in studying workplace learning: strengths and limitations of the adopted approach. Paper for BERA Annual Conference.

Stenström, M.L. \& Tynyälä, P. (eds.)(2009). Towards integration of work and learning. Dordrecht: Springer.

Teitelbaum, K. (2017). Citizenship, workers education and radical activism in early 20th century United States. En F. Marhuenda. Vocational education beyond skill formation: VET between civic, industrial and market tensions. Bern: Peter Lang, pp. 109-138.

\section{Agradecimientos}

Agradezco la revisión de versiones anteriores de este texto, de miembros del equipo de investigación, en particular de José Luis Belver y Alicia Ros. 
